\title{
Real-time Data Classification for EEG and EKG Analysis Based on Artifacts Detection
}

\author{
Zhanfeng $\mathrm{Ji}^{1,2}$, Takenao Sugi ${ }^{1}$, Satoru Goto ${ }^{1}$, Xingyu Wang ${ }^{2}$, and Masatoshi Nakamura ${ }^{3}$ \\ ${ }^{1}$ Dept. of Advanced System Control Engineering, Saga University \\ 1 Honjo-machi, Saga 840-8502, Japan \\ ${ }^{2}$ Department of Automation, East China University of Science and Technology \\ 130 Road Meilong, Shanghai 200237, China \\ ${ }^{3}$ Research Institute of Systems Control Institute for Advanced Research and Education, Saga University \\ Yoka-machi, Saga 840-0047, Japan \\ E-mail:jzf1228@gmail.com
}

\begin{abstract}
Electroencephalogram (EEG) and electrocardiogram (EKG) signals are usually contaminated by artifacts. Characteristic analysis based on contaminated signals would lead to spurious results. In order to extract reliable characteristics from EEG and EKG signals, it is necessary to select available signals. In this paper, a real-time data classification system for EEG and EKG analysis was developed. Automatic artifacts detection is helpful for checking long-time recording, and real time classification is useful to obtain satisfactory recording and better understanding of artifacts.
\end{abstract}

\section{Introduction}

Electroencephalogram (EEG) and electrocardiogram (EKG) are important for clinical diagnosis. They have been widely studied in hospitals and laboratories, including patients and healthy subjects.

EEG analysis is useful in a lot of research fields, such as infants monitoring, polysomnography (PSG) analysis, brain computer interface, Parkinson diagnosis, and epilepsy diagnosis. In case of EKG analysis, heart rate variability (HRV) analysis has become a popular tool to investigate autonomous nervous system and to predict cardiac diseases [1].

For reliable analysis, only available signals should be used. EEG signals are susceptible to kinds of artifacts, such as blink, muscle noise, electrode artifact. EKG signals are also often distorted by physical activities or other artifacts. Characteristic analysis based on contaminated signals would lead to spurious results.

Long-time recordings are useful in clinical diagnosis, such as sleep stage analysis, monitoring in the intensive care unit (ICU). For short time EEG analysis, available signals can be selected by visual inspection [2]. But for long time recording, it is time consuming for experts to check artifacts manually. On the other hand, visual inspection is difficult for unskilled researchers. In order to obtain reliable results, and reduce the heavy burden of experts, automatic artifacts detection and data classification for EEG and EKG are necessary.

A few papers related to artifacts detection for EEG and EKG signals were reported. An automatic artifact removal method for EEG analysis during long-term recording was reported [3]. The segments which might include artifacts were removed. An automatic artifact detection system for EEG signals was reported in [4]. However, the artifacts were not discriminated in details in these studies. An automatic detection method for HRV analysis was reported in [5]. The method only considered RR interval series.

In previous work [6], an artifacts detection method was developed and the vigilance level was evaluated, in order to acquire satisfactory EEG recording under awaking status with eyes closed. In this paper, a real-time data classification method based on artifacts detection was developed, in order to obtain meaningful analysis of EEG and EKG signals, by rejection of contaminated signals.

\section{Method}

\subsection{Subjects and Data Acquisition}

The EEG signals were recorded on 8 healthy subjects, aged 22-25 years old, in systems control laboratory, Saga University of Japan. The subjects were settled in a quiet, electrical shielding room.

EEG electrodes were placed on the scalp according to the international 10-20 system at the following areas: $F_{P_{1}}$, $\mathrm{F}_{3}, \mathrm{O}_{1}, \mathrm{~F}_{\mathrm{P} 2}, \mathrm{~F}_{4}, \mathrm{O}_{2}, \mathrm{~F}_{\mathrm{Z}}$, and $\mathrm{C}_{\mathrm{Z}}$, which were referred to the ipsilateral ear electrode $\left(A_{1}\right.$ or $\left.A_{2}\right) . F_{Z}$ and $C_{Z}$ were referred to the average of all the electrodes $\left(A_{a v}\right)$. The EEG signals were recorded using $\mathrm{Ag} / \mathrm{AgCl}$ electrodes.

In case of EKG recording, in order to obtain the largest amplitude of $\mathrm{R}$ peak, a pair of electrodes for one channel was placed according to standard limb lead II.

The EEG and EKG signals were recorded on a digital EEG machine (Nihon-Koden EEG2110) with the sampling frequency of $200 \mathrm{~Hz}$ and the upper frequency cut-off at 60 $\mathrm{Hz}$, and transferred to the real-time data classification system through a $\mathrm{DA}-\mathrm{AD}$ converter. The results were 
displayed and updated in real-time. An executant was asked to monitor the results and to assign tasks to subjects. Tasks of continuous long-time mental calculation and relaxation with music were performed.

\subsection{Real-time Data Classification System}

The hardware connection of digital EEG machine and real-time system is shown in Fig.1 (a). EEG signals (channel 0-7) were transferred with a time constant (TC) of $0.3 \mathrm{~s}$, and EKG signals were transferred with a TC of $2.0 \mathrm{~s}$ (channel 8) and a TC of $0.03 \mathrm{~s}$ (channel 9). The PC for real-time data classification system was a Toshiba notebook with a windows vista system, a $2.1 \mathrm{GHz}$ processor and 2 GB RAM. The real-time system was programmed with Microsoft Visual $\mathrm{C}++6.0$. The interface is shown in Fig.1 (b). Part A displayed the original waveforms, and part B displayed the results of artifacts detection and data classification.

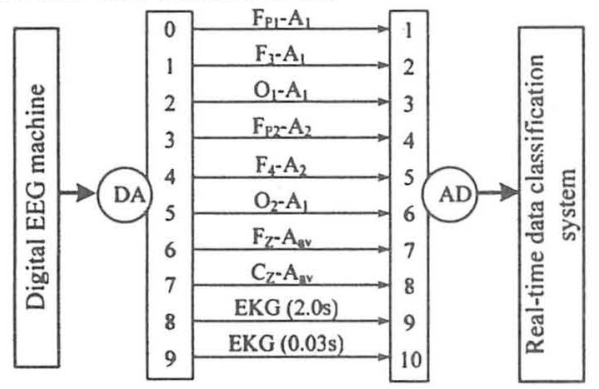

(a) Hardware connection

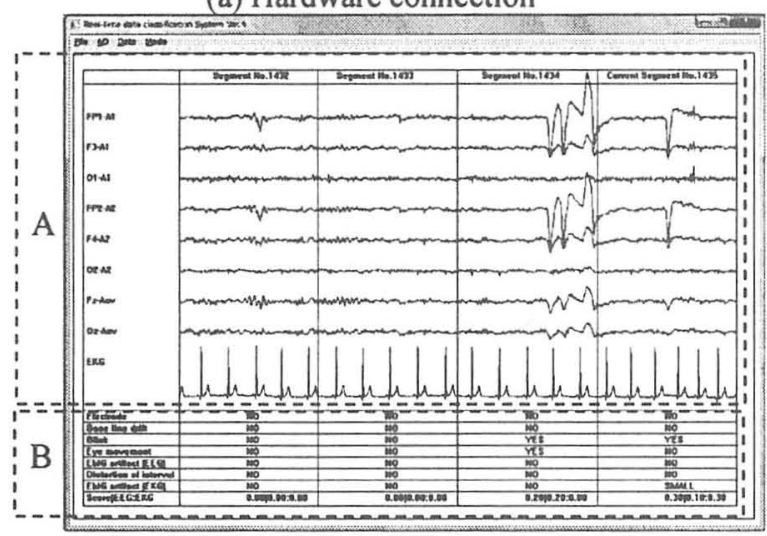

(b) Windows interface

Fig.1 Real-time data classification system

\begin{tabular}{|l|l|}
\hline \multicolumn{2}{|c|}{ Segment data acquisition } \\
\begin{tabular}{|l|l|}
\hline Artifacts in EEG \\
(1) Blink \\
(2) Ear-lobe reference \\
electrode artifact \\
(3) Base-line drift \\
(4) Eye movement \\
(5) EMG
\end{tabular} & $\begin{array}{l}\text { Artifacts in EKG } \\
\text { (1) Distortion of } \\
\text { interval } \\
\text { (2) EMG }\end{array}$ \\
\hline \multicolumn{2}{|c|}{ Data classification } \\
\hline
\end{tabular}

Fig.2 Flowchart of data classification for one segment
The recording was divided into $5.12 \mathrm{~s}$ segments. The results were updated after each segment. The procedure of data classification for each segment is shown in Fig.2.

\subsection{Definition of Parameters}

Several parameters are employed for artifacts detection. The definition of these parameters is given in Table 1 .

Parameters for EEG include the amplitude $A_{\mathrm{z}}(x)$, symmetry $P_{\mathrm{z}}(x, y)$, extension $E_{\mathrm{z}}(x, y)$, and correlation coefficient $R(x, y)$, where both $x$ and $y$ represent specific electrodes of $\mathrm{F}_{\mathrm{P} 1}, \mathrm{~F}_{3}, \mathrm{O}_{1}, \mathrm{~F}_{\mathrm{P} 2}, \mathrm{~F}_{4}, \mathrm{O}_{2}, \mathrm{~F}_{\mathrm{Z}}$, and $\mathrm{C}_{\mathrm{Z}} ; z$ denotes respective EEG component: $L(0-0.5 \mathrm{~Hz}), \delta(0.5-4 \mathrm{~Hz})$, $\theta(4-8 \mathrm{~Hz}), \quad \alpha(8-13 \mathrm{~Hz}), \quad \beta(13-25 \mathrm{~Hz}), \quad H(35-50 \mathrm{~Hz})$. The following items are employed in the definition:

- $S_{\mathrm{Z}}(x)$ is the summation of periodogram with the frequency band of $z$ in channel $x$;

- $S_{\mathrm{Z}}(x-y)$ is the summation of periodogram with the frequency band of $z$ in channel $x$ and $y$, in which the EEG time series of channel $y$ is subtracted from that of channel $x$;

- $S_{\mathrm{Z}}(x+y)$ is the summation of periodogram with the frequency band of $z$ in channel $x$ and $y$, in which the EEG time series of channel $y$ adds that of channel $x$.

Parameters for EKG include the RR interval series $y(n)$, estimated base-line drift $E_{\mathrm{BLD}}$, and the amplitude $A_{\mathrm{H} 2}$, where $x(n)$ represent the estimated base-line of EKG signals with a TC of 2.0 s, by a discrete Fourier transform (DFT) low pass filter with a upper cut-off frequency of $1 \mathrm{~Hz} ; \mathrm{H} 2$ denotes high frequency band of $(45-59 \mathrm{~Hz})$ and $(61-100 \mathrm{~Hz})$; and $S_{\mathrm{H} 2}$ is the summation of frequency component of $\mathrm{H} 2$ for EKG signals with a TC of 2.0 s.

\begin{tabular}{|c|c|c|}
\hline & Symbols & Description and formula \\
\hline \multirow{4}{*}{ EEG } & $A_{\mathrm{Z}}(x)$ & Amplitude $(\mu \mathrm{V}): 6 \sqrt{S_{2}(x)}$ \\
\hline & $P_{\mathrm{Z}}(x, y)$ & Symmetry $(\%): \quad 6 \sqrt{S_{z}(x-y)} / 6 \sqrt{S_{z}(x+y)} \times 100$ \\
\hline & $E_{\mathrm{Z}}(x, y)$ & Extension $(\%): 6 \sqrt{S_{z}(y)} / 6 \sqrt{S_{z}(x)} \times 100$ \\
\hline & $R(x, y)$ & Correlation coefficient of channel $x$ and $y$ \\
\hline \multirow{3}{*}{ EKG } & $y(n)$ & $\mathrm{RR}$ interval series \\
\hline & $E_{\mathrm{BLD}}$ & $\begin{array}{l}\text { Estimated base-line drift : } \\
\qquad E_{\mathrm{BDD}}=\max \{x(n)\}-\min \{x(n)\}\end{array}$ \\
\hline & $A_{\mathrm{H} 2}$ & Amplitude $(\mu \mathrm{V}): 6 \sqrt{S_{\mathrm{H} 2}}$ \\
\hline
\end{tabular}

\subsection{Artifacts Detection}

2.4.1 Artifacts in EEG. EEG recording is often contaminated by loose electrode, which may result in electrode problem or base-line drift. In this paper, the electrode artifact indicates the ear-lobe reference electrode artifact. When the waveforms are similar in all the channels on the same site and different with the other site, the ear-lobe reference electrode artifact is detected [2]. The base-line drift artifact is also a type of electrode artifact. The impedance variety of electrodes is the main reason, which may be brought by body movement or sweating. When the waveform is similar in all the channels on both site of the scalp, the base-line drift artifact is detected.

On the other hand, EEG recording is also often 
contaminated by physical activities, such as blink, eye movement, and electromyography (EMG) activity. These artifacts have effect on EEG components.

The criteria for artifacts detection are illustrated by the equations in Table 2.

\begin{tabular}{ll} 
Table 2 Criteria for artifacts in EEG \\
\hline Artifact & Judgment conditions \\
\hline Electrode & Left: $A_{\delta}(x) \geq 25 \mu \mathrm{V}$, \\
& $\min \left\{R\left(F_{\mathrm{P} 1}, F_{3}\right), R\left(O_{1}, F_{3}\right), R\left(O_{1}, F_{\mathrm{P} 1}\right)\right\}>0.9$, \\
& $\operatorname{Right:} A_{\delta}(x) \geq 25 \mu \mathrm{V}$, \\
& $\min \left\{R\left(F_{\mathrm{P}}, F_{4}\right), R\left(O_{2}, F_{4}\right), R\left(O_{2}, F_{\mathrm{P} 2}\right)\right\}>0.9$, \\
& $\min \left\{R\left(F_{\mathrm{P} 1}, F_{\mathrm{P} 2}\right), R\left(F_{3}, F_{4}\right), R\left(O_{1}, O_{2}\right)\right\}<0.8$. \\
\hline Base-line drift & $\min \left\{A_{\mathrm{L}}(x)\right\} \geq 60 \mu \mathrm{V}$, \\
& $\min \left\{R\left(F_{\mathrm{P} 1}, F_{\mathrm{P} 2}\right), R\left(F_{3}, F_{4}\right), R\left(O_{1}, O_{2}\right)\right\}>0.8$ \\
\hline Blink & Existence of $\delta$ component: \\
& $A_{\delta}\left(F_{\mathrm{P} 1}\right)>40 \mu \mathrm{V}, A_{\delta}\left(F_{\mathrm{P} 2}\right)>40 \mu \mathrm{V}$ \\
& Symmetry of the waveform: \\
& $A_{\delta}\left(F_{\mathrm{P} 1}+F_{\mathrm{P} 2}\right)>80 \mu \mathrm{V}, P_{\delta}\left(F_{\mathrm{P} 1}, F_{\mathrm{P} 2}\right)<55 \%$ \\
& Extention to central region: \\
& $E_{\delta}\left(F_{\mathrm{P} 1}, F_{3}\right) \leq 85 \%, E_{\delta}\left(F_{\mathrm{P} 2}, F_{4}\right) \leq 85 \%$ \\
& Existence of $L$ component: \\
& $A_{\mathrm{L}}\left(F_{\mathrm{P} 1}\right)>60 \mu \mathrm{V}, A_{\mathrm{L}}\left(F_{\mathrm{P} 2}\right)>60 \mu \mathrm{V}$, \\
& Symmetry of the waveform: \\
& $A_{\mathrm{L}}\left(F_{\mathrm{P} 1}+F_{\mathrm{P} 2}\right)>120 \mu \mathrm{V}, P_{\mathrm{L}}\left(F_{\mathrm{P} 1}, F_{\mathrm{P} 2}\right)<55 \%$, \\
& Extention to central region: \\
& $E_{\mathrm{L}}\left(F_{\mathrm{P} 1}, F_{3}\right) \leq 85 \%, E_{\mathrm{L}}\left(F_{\mathrm{P} 2}, F_{4}\right) \leq 85 \%$, \\
& $A_{\mathrm{H}}(x) \geq 10 \mu \mathrm{V}, A_{\mathrm{H}}(x) \geq A_{\beta}(x)$ \\
\hline Eye movement & \\
\hline EMG &
\end{tabular}

2.4.2 Artifacts in EKG. The artifacts detection method was proposed for heart rate variability analysis under quiet status. The artifacts including distortion of RR interval and EMG artifacts were considered in this paper.

As shown in Table 3, distortion of RR interval is classified as SERIOUS and MEDIUM. If the $R$ peak is missed or wrongly detected, it is defined as SERIOUS. If large base-line drift is detected, but the $R$ peaks are detected correctly, it is defined as MEDIUM. Since continuous RR intervals are necessary for HRV analysis, EKG signals are unavailable when $R$ peak can't be detected. As shown in Table $3, y(i)$ indicates RR interval series, which is always shorter than $1.2 \mathrm{~s}$, and longer than $0.4 \mathrm{~s}$, when the subject is quiet. If $y(i)>1.2 \mathrm{~s}$ is detected, $\mathrm{R}$ peaks may be missed; and if $y(i)<0.4 \mathrm{~s}$ is detected, artifacts may be mistook as $\mathrm{R}$ peak.

As for EMG artifacts, large ones will lead to wrong detection of $\mathrm{R}$ peak, and small ones could reflect the body movement. The criteria for artifacts detection are illustrated by the equations in Table 3 .

\begin{tabular}{ll}
\multicolumn{2}{c}{ Table 3 Criteria for artifacts in EKG } \\
\hline Artifact & Judgment conditions \\
\hline Distortion of & SERIOUS: $y(i)>1.2 \mathrm{~s}$, or $y(i)<0.4 \mathrm{~s}$ \\
\cline { 2 - 2 } interval & MEDIUM: $E_{\mathrm{BLD}}>900 \mu \mathrm{V}$ \\
\hline EMG artifacts & LARGE: $A_{\mathrm{Hz}}(x) \geq 200 \mu \mathrm{V}$ \\
\cline { 2 - 2 } & SMALL: $A_{\mathrm{Hz}}(x) \geq 58 \mu \mathrm{V}$ \\
\hline
\end{tabular}

\subsection{Data Classification}

Weight coefficients were given to the artifacts for evaluation of the quality of each segment, as shown in
Table 4. Then the segments were scored on the basis of detected artifacts.

The evaluation for EEG and EKG is independent. The score is equal to the summation of the weight of detected artifacts. The higher score was defined as the final score. 0 indicates the best signals, and 1 indicates the worst.

For EMG artifacts in EEG, the score is defined as $0.6^{*} \mathrm{~m} / \mathrm{n}$, where $m$ indicates the amount of contaminated channels, and $n$ indicates the amount of total channels. For EKG evaluation, if the score is larger than 1 , it will be scored as 1 .

\begin{tabular}{lll}
\multicolumn{2}{c}{ Table 4 Weight coefficient } \\
\hline & Artifacts & Weights \\
\hline \multirow{3}{*}{ EEG } & Electrode & 0.1 \\
& Base-line drift & 0.1 \\
& Blink & 0.1 \\
& Eye movement & 0.1 \\
& EMG artifacts & 0.6 \\
\hline \multirow{2}{*}{ EKG } & Distortion of RR interval & 1 (SERIOUS) \\
& & 0.5 (MEDIUM) \\
& EMG artifacts & 1 (LARGE) \\
& & 0.3 (SMALL) \\
\hline
\end{tabular}

The following parameters are selected to evaluate the effectiveness of data classification: the amplitude of $\alpha$ band $\left(A_{\alpha}\right)$ and the amplitude of $\beta$ band $\left(A_{\beta}\right)$ related to EEG analysis, heart rate (HR), power of low frequency components (LF) and high frequency components (HF) related to EKG analysis.

Three continuous segments (about 16s) were employed for EKG analysis. RR interval series were reconstructed with linear interpolation method and a sampling frequency of $0.5 \mathrm{~Hz}$. LF and HF were calculated by the spectrum of maximum entropy method (MEM) with a resolution of $0.01 \mathrm{~Hz}$, and a maximum order of 20 . For comparison, the parameters were also calculated by the spectrum of FFT.

\section{Results}

\subsection{Artifact Detection}

The recording of 8 subjects were detected, 15856 segments in total, 1982 segments for each subject. As a result, for detection of artifacts in EEG, 37 segments $(0.23 \%)$ were detected for electrode artifacts, 11 segments $(0.07 \%)$ for base-line drift, 13891 segments $(87.61 \%)$ for blink, 7741 segments $(48.82 \%)$ for eye movement, 8565 segments (54.02\%) for EMG artifacts; and for detection of artifacts in EKG, 1426 segments (8.99\%) were detected for distortion of RR interval, 10053 segments $(63.40 \%)$ for EMG artifacts.

An example of real-time data classification is shown in Fig.3. Four continuous segments are displayed.

The results of data classification are displayed under the original waveforms. The notation "YES" indicates that artifacts are detected, while the notation "NO" indicates no artifact. In case of EMG artifacts in EEG, the following number indicates the amount of contaminated electrodes. The notation "SERIOUS", "MEDIUM", "LARGE", and "SMALL" are employed to display the degree of detected EKG artifacts. 


\subsection{Data Classification}

The quality of the signals is evaluated by detected artifacts and the weight coefficients. The scores are shown in the last line. The scores for EEG and EKG are also displayed, as shown in Fig.3.

If the scores of three continuous segments are lower than 0.2 , the last segment will be marked with $\mathrm{A}$, which stands for the clear segment. If the score of a certain segment is higher than or equal to 0.8 , the segment wirk be marked with $B$, which stands for the serious contaminated segment. As a result of one subject, 141 segments were marked with $\mathrm{A}$, and 46 segments were marked with $\mathrm{B}$.

The averaged $A_{\alpha}$ and $A_{\beta}$ for selected segments are shown in Table 5 . In case of $A_{a}, \mathrm{~F}_{\mathrm{P} 1}$ and $\mathrm{F}_{\mathrm{P} 2}$ were obviously affected. In case of $A_{\beta}$, obvious variation was observed at all the channels.

\begin{tabular}{ccccccccc}
\multicolumn{10}{c}{ Table 5 EEG analysis } \\
\hline$A_{a}(\mu \mathrm{V})$ & $\mathrm{F}_{\mathrm{P} 1}$ & $\mathrm{~F}_{3}$ & $\mathrm{O}_{1}$ & $\mathrm{~F}_{\mathrm{P} 2}$ & $\mathrm{~F}_{4}$ & $\mathrm{O}_{2}$ & $\mathrm{~F}_{\mathrm{Z}}$ & $\mathrm{C}_{\mathrm{Z}}$ \\
\hline $\mathrm{A}$ & 12.33 & 11.17 & 12.93 & 11.69 & 11.64 & 10.92 & 12.88 & 14.35 \\
$\mathrm{~B}$ & 22.97 & 15.92 & 17.71 & 20.30 & 14.74 & 13.72 & 14.20 & 13.57 \\
\hline$A_{\mathrm{P}}(\mu \mathrm{V})$ & $\mathrm{F}_{\mathrm{P} 1}$ & $\mathrm{~F}_{3}$ & $\mathrm{O}_{1}$ & $\mathrm{~F}_{\mathrm{P} 2}$ & $\mathrm{~F}_{4}$ & $\mathrm{O}_{2}$ & $\mathrm{~F}_{\mathrm{Z}}$ & $\mathrm{C}_{\mathrm{Z}}$ \\
\hline $\mathrm{A}$ & 8.65 & 8.19 & 14.96 & 7.05 & 7.12 & 9.75 & 6.92 & 7.55 \\
$\mathrm{~B}$ & 22.36 & 30.82 & 28.71 & 19.18 & 21.05 & 20.14 & 16.14 & 15.59 \\
\hline
\end{tabular}

The averaged HR, LF, and HF for selected segments are shown in Table 6 . The segments including miss detection or false detection of $\mathrm{R}$ peak were excluded. The results showed that the values of the parameters for group B were obviously higher than that for group A.

\begin{tabular}{rrrrrr}
\multicolumn{6}{c}{ Table 6 EKG analysis } \\
\hline \multirow{2}{*}{$\mathrm{HR}(/ \mathrm{min})$} & \multicolumn{2}{c}{ MEM } & \multicolumn{2}{c}{ FFT } \\
\cline { 3 - 6 } & & $\mathrm{LF}\left(\mathrm{ms}^{2}\right)$ & $\mathrm{HF}\left(\mathrm{ms}^{2}\right)$ & $\mathrm{LF}\left(\mathrm{ms}^{2}\right)$ & $\mathrm{HF}\left(\mathrm{ms}^{2}\right)$ \\
\hline A & 60.80 & 31.21 & 39.31 & 37.18 & 50.09 \\
B & 71.58 & 147.47 & 55.47 & 245.78 & 140.75 \\
\hline
\end{tabular}

\section{Discussion}

\subsection{Weight Coefficients}

4.1.1 Weight setting. The quality of recording is important for obtaining reliable result. The purpose of this research was to study the relationship between fatigue level and bio-medical characteristics. Therefore, it is necessary to consider all the artifacts in EEG and EKG signals. The weight of artifacts was given to each artifact according to the importance level of the contaminated information.

In case of EEG analysis, frequency components of $\delta, \theta$, $\alpha$, and $\beta$ are usually studied. In most researches, the information of $\alpha$ band and $\beta$ band is more important than that of $\delta$ band and $\theta$ band.

The EMG artifacts increase the power of $\beta$ band obviously and sometimes affect $\alpha$ band. So that a high weight coefficient of 0.6 was given.

The ear-lobe reference electrode artifact, the base-line drift, blink, and eye movement usually increase the power spectrum of the low frequency components, and only affect the power of $\delta$ band, sometimes $\theta$ band. Moreover, blink and eye movement can be frequently encountered in EEG recording, with an experimental condition of eyes open. Both of them usually only affect the frontal electrodes. Therefore these four artifacts were given a low weight coefficient of 0.1 .

In case of EKG analysis, accuracy detection of $\mathrm{R}$ peak is the most important. Both SERIOUS distortion of RR interval and LARGE EMG artifacts can result in miss detection or false detection of $\mathrm{R}$ peak. In this case, the signals are unavailable for HRV analysis, so that a reject coefficient of 1 was given. As for MEDIUM distortion of RR interval and SMALL EMG artifacts, they only distort the waveform of EKG signals, but $R$ peak is still clear. Both of them may reflect the degree of body movement. However, MEDIUM distortion of RR interval is caused by large base-line drift of EKG signals, which may affect RR interval more than SMALL EMG artifacts. Therefore, a medium weight coefficient of 0.5 was given to MEDIUM distortion of RR interval, and a low weight coefficient of 0.3 was given to SMALL EMG artifacts.

4.1.2 Extended application. In this paper, the segment score is defined as the higher score of EEG and EKG, because both EEG and EKG signals are important.

Users should define the segment score according to their specific research purposes. Similarly, users should also adjust the weight coefficients according to the influence of artifacts in their analysis.

For example, if the purpose is to analyze the characteristics of EEG, a weight of 0 should be given to the artifacts in EKG.

Besides, the electrodes for EEG recording in this paper are only the necessary electrodes. If users employ other electrodes, EMG artifacts should be detected for additional channels.

\subsection{Effectiveness of Data Classification}

The artifacts detection method was established taking account on visual inspection, considering the power of frequency bands and the characteristics of multi-channel waveforms. The thresholds were determined by using training data, including 80 typical segments which consist of contaminated signals and clear signals from the original recording.

As shown in Fig.3, the artifacts were detected correctly with real-time data classification system, and the score was properly given.

As shown in Table 5 and Table 6, the difference between the best segments (A) and the worst segments (B) is obvious. If the worst segments were considered for EEG or EKG analysis, the results of characteristic analysis would be unreliable.

\subsection{Real-time Application}

Artifacts detection is crucial for reliable analysis of EEG or EKG signals. However, since the artifacts are usually detected after recording; it is very difficult to find out the 
actual reason. In this paper, the data classification method is developed for real-time application. It is convenient to find out the reason of artifacts during recording process.

As mentioned in section 2.2 , a $2.1 \mathrm{GHz}$ processor was employed, and the total processing time for each segment is within $0.1 \mathrm{~s}$. The performance is enough for real-time application.

\section{Conclusion}

The real-time data classification method proposed in this paper is developed to select available signals for EEG and EKG analysis. The method is effective to reduce the burden of experts and useful to help unskilled researchers to avoid spurious results. Real-time application is propitious to obtain satisfactory recording and better understanding of artifacts.

\section{References}

[1] Task Force of the European Society of Cardiology and the North American Society of Pacing and

Electrophysiology: Heart rate variability: Standards of measurement, physiological interpretation, and clinical use, Eur. Heart J., 17, pp. 354-381, 1996.

[2] L Pezard, R Jech, E Ruzicka: Investigation of non-linear properties of multichannel EEG in the early stages of Parkinson's disease, Clin. Neu., 112, pp.38-45, 2001.

[3] R Agarwal, J Gotman, D Flanagan, B Rosenblatt: Automatic EEG analysis during long-term monitoring in the ICU, Electroencephalogr. Clin. Neurophysiol., 107, pp.44-58, 1998.

[4] P J Durka, H Klekowicz, K J Blinowska, W Szelenberger, S Niemcewicz: A simple system for detection of EEG artifacts in polysomnographic recordings, IEEE Biomed., 50, pp.526-528, 2003.

[5] X Xu, S Stephanie, CHIME Study Group: Automatic detection of artifacts in heart period data, J. Elcardiol., 34, pp.205-210, 2001.

[6] M Nakamura, Q Chen, T Sugi, A Ikeda, H Shibasaki: Technical quality evaluation of EEG recording based on electroencephalographer s' knowledge, Med. Eng. Phy., 27, pp.93-100, 2005.

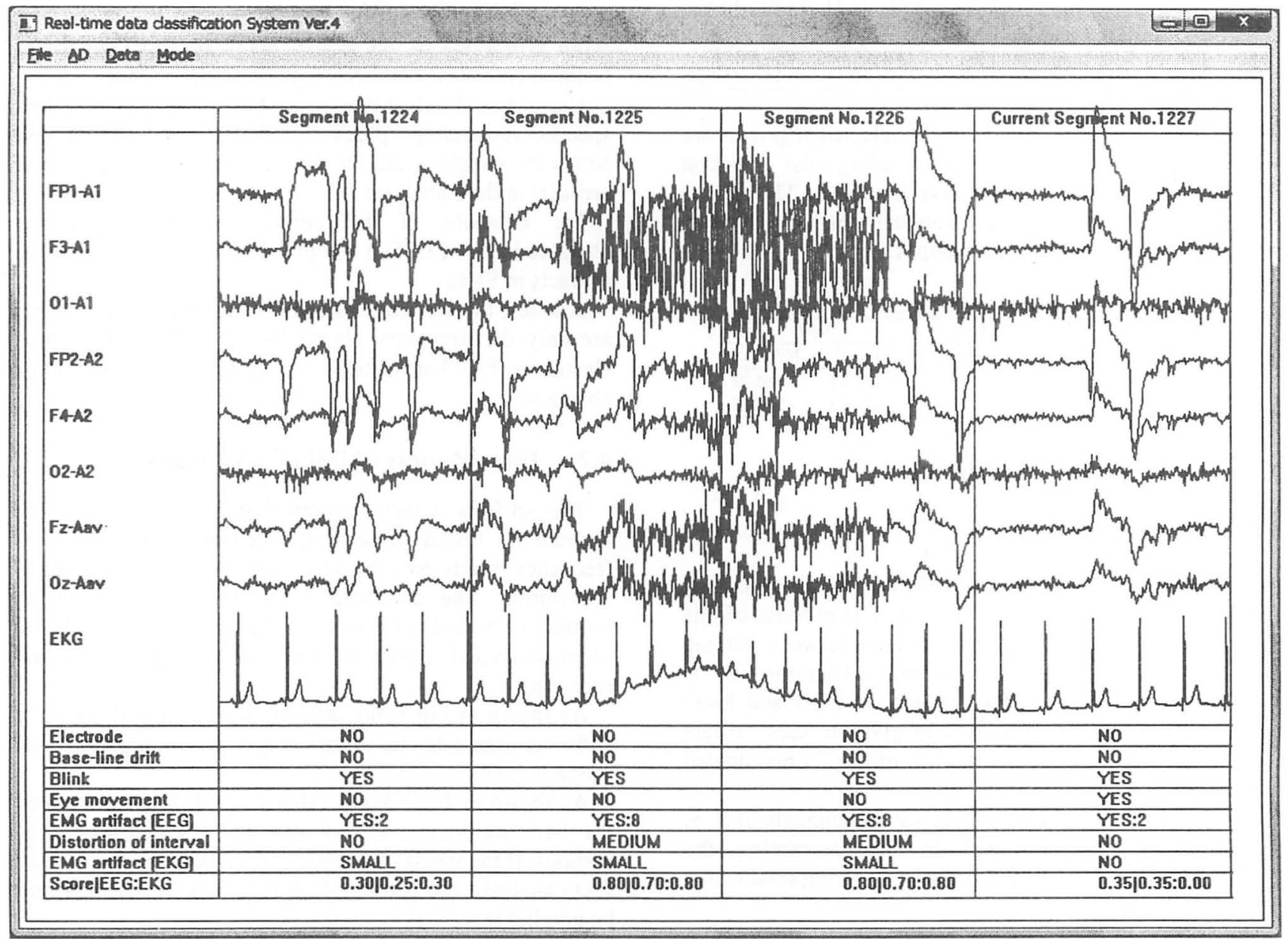

Fig.3 An example of real-time data classification of four continuous segments 